\title{
Epigenetic clock and DNA methylation analysis of porcine models of aging and obesity
}

\author{
Kyle M. Schachtschneider • Lawrence B. Schook • Jennifer J. Meudt • \\ Dhanansayan Shanmuganayagam · Joseph A. Zoller • Amin Haghani • \\ Caesar Z. Li · Joshua Zhang · Andrew Yang $\cdot$ Ken Raj · Steve Horvath
}

Received: 18 May 2021 / Accepted: 9 August 2021 / Published online: 15 September 2021

(C) The Author(s) 2021

\begin{abstract}
DNA-methylation profiles have been used successfully to develop highly accurate biomarkers of age, epigenetic clocks, for many species. Using a custom methylation array, we generated DNA methylation data from $n=238$ porcine tissues including blood, bladder, frontal cortex, kidney, liver, and lung, from domestic pigs (Sus scrofa domesticus) and minipigs (Wisconsin Miniature Swine ${ }^{\mathrm{TM}}$ ). Samples used in this study originated from Large White $\mathrm{X}$ Landrace crossbred pigs, Large White X Minnesota minipig crossbred pigs, and Wisconsin Miniature
\end{abstract}

Supplementary Information The online version contains supplementary material available at https://doi. org/10.1007/s11357-021-00439-6.

K. M. Schachtschneider · L. B. Schook

Department of Radiology, University of Illinois At

Chicago, Chicago, IL, USA

e-mail: kschach2@uic.edu

L. B. Schook

e-mail: schook@illinois.edu

K. M. Schachtschneider

Department of Biochemistry and Molecular Genetics,

University of Illinois At Chicago, Chicago, IL, USA

K. M. Schachtschneider

National Center for Supercomputing Applications,

University of Illinois At Urbana-Champaign, Urban, IL, USA

L. B. Schook

Department of Animal Sciences, University of Illinois At

Urbana-Champaign, Urbana, IL, USA
Swine ${ }^{\mathrm{TM}}$. We present 4 epigenetic clocks for pigs that are distinguished by their compatibility with tissue type (pan-tissue and blood clock) and species (pig and human). Two dual-species human-pig pan-tissue clocks accurately measure chronological age and relative age, respectively. We also characterized CpGs that differ between minipigs and domestic pigs. Strikingly, several genes implicated by our epigenetic studies of minipig status overlap with genes (ADCY3, TFAP2B, SKOR1, and GPR61) implicated by genetic studies of body mass index in humans. In addition, CpGs with different levels of methylation between the two pig breeds were identified proximal to genes involved in blood LDL levels and cholesterol

\author{
J. J. Meudt · D. Shanmuganayagam \\ Biomedical \& Genomic Research Group, Department \\ of Animal and Dairy Sciences, University of Wisconsin - \\ Madison, Madison, WI, USA \\ e-mail: jjmeudt@wisc.edu \\ D. Shanmuganayagam \\ e-mail: dshanmug@wisc.edu \\ D. Shanmuganayagam \\ Department of Surgery, University of Wisconsin School \\ of Medicine and Public Health, Madison, WI, USA \\ J. A. Zoller · A. Haghani - C. Z. Li · A. Yang · \\ S. Horvath $(\square)$ \\ Department of Biostatistics, Fielding School of Public \\ Health, University of California, Los Angeles, \\ Los Angeles, CA, USA \\ e-mail: shorvath@mednet.ucla.edu
}


synthesis, of particular interest given the minipig's increased susceptibility to cardiovascular disease compared to domestic pigs. Thus, breed-specific differences of domestic and minipigs may potentially help to identify biological mechanisms underlying weight gain and aging-associated diseases. Our porcine clocks are expected to be useful for elucidating the role of epigenetics in aging and obesity, and the testing of anti-aging interventions.

Keywords Porcine $\cdot$ Pig $\cdot$ Minipigs $\cdot$ Aging · Development $\cdot$ Epigenetic clock $\cdot$ DNA methylation

\section{Introduction}

Pigs (Sus scrofa) are omnivores that last shared a common ancestor with humans between 79 and 97 million years ago $[1,2]$. The domestication of pigs dates back to approximately 10,000 years, where they were domesticated from local wild boars across Eurasia [3, 4]. Since then, a wide variety of domestic and minipig breeds have been selectively bred for agricultural and biomedical purposes. While murine models have been traditionally used in biomedical research, there are added advantages to the use of porcine models in translational research. This includes their comparable size, anatomy, physiology, immunology, metabolism, and genetics with humans [5-7]. At the cellular levels, we have previously demonstrated similar genome-wide DNA methylation patterns between

\author{
A. Haghani \\ e-mail: ahaghani@g.ucla.edu \\ C. Z. $\mathrm{Li}$ \\ e-mail: lizexuan@g.ucla.edu \\ A. Yang \\ e-mail: andy.yang@yale.edu \\ J. Zhang $\cdot S$. Horvath \\ Department of Human Genetics, David Geffen School \\ of Medicine, University of California, Los Angeles, Gonda \\ Building, 695 Charles Young Drive South, Los Angeles, \\ CA 90095, USA \\ e-mail: joshuazhang@g.ucla.edu \\ K. Raj \\ Radiation Effects Department, Centre for Radiation, \\ Chemical and Environmental Hazards, Public Health \\ England, Chilton, Didcot, UK \\ e-mail: ken.raj@phe.gov.uk
}

pigs and humans across a range of biomedically relevant tissues [8, 9], further supporting the high relevance of pigs in modeling human disease and development. Indeed, these advantages have been recognized and porcine models are already being used in biomedical research [10-19]. However, the large size and low propensity for atherosclerosis development in particular [20], limited the use domestic pigs as cardiovascular models. To overcome these issues, selectively bred and genetically modified minipigs have emerged over recent years as excellent models of hypercholesterolemia, atherosclerosis, metabolic syndrome, diabetes, and even cancer [13, 21-27]. Due to their smaller size, ease of handling, and genetic manipulability, minipigs are becoming increasingly important animal models for a wide range of human pathologies [12].

The study of any disease would be incomplete without an understanding of how age contributes to the malfunctioning of cells, tissues and organs. Despite this acknowledgement, the contribution of age to pathology has been largely unaddressed, not for the lack of will, but means. In the absence of an accurate way to quantify biological age, time (chronological age) is adopted as a surrogate that is manifestly unsatisfactory, as it remains unresponsive to biological fitness or frailty. The need for a measure of age that is based on biology is clear, and hints that this may be possible emerged when DNA methylation level was observed to change with advancing age. DNA methylation is an epigenetic modification that controls gene expression. The significance of its age-associated change was a subject of speculation until recently, when an array-based technology was developed to accurately measure its level on specific cytosine-phosphate-guanines (CpGs) in the genome. DNA methylation levels allow one to build age estimators (pan tissue clocks) that apply to most cells of the human body [28]. The rate of epigenetic aging was observed to be associated with a wide range of human conditions including mortality risk, Down syndrome, HIV infection and obesity [29-34], indicating that epigenetic age is a measure, at least to some degree, of biological age.

It is evident that the extrapolation of this epigenetic clock to other species, especially those such as pigs that are employed as disease models in biomedical research, will greatly facilitate research into the influence of age on pathology. This would also permit the 
quantitative testing of interventions that could potentially mitigate age effects on pathology. Towards this end, we aimed to develop epigenetic clocks that apply to both humans and pigs at the same time. To overcome the species barrier, we used a DNA methylation array platform (HorvathMammalMethylChip40) that encompasses $\mathrm{CpGs}$ flanked by DNA sequences that are conserved across different species of the mammalian class.

Here, we present highly accurate epigenetic clocks that apply to both humans and different pig breeds: the regular sized domestic pigs, Wisconsin Miniature Swine ${ }^{\mathrm{TM}}$, and a cross between domestic and Minnesota minipigs. The human-pig clock provides increases the probability that findings in pigs will translate to humans, and vice versa. We also characterized (1) age-related changes in the porcine methylome and (2) cytosines that differ between minipigs and regular sized pigs.

\section{Methods}

Porcine samples

All animal procedures were approved by the University of Illinois and University of Wisconsin Institutional Animal Care and Use Committee, and all animals received humane care according to the criteria outlined in the Guide for the Care and Use of Laboratory Animals. Porcine whole blood samples $(n=146)$ were collected from female Large White X Landrace crossbred domestic pigs $(n=84$, age range 11-2285 days) and Wisconsin Miniature Swine ${ }^{\mathrm{TM}}$ ( $n=60$, age range $8-1880$ days) at the University of Wisconsin-Madison. Whole blood $(n=16)$ and tissue samples (bladder, frontal cortex, kidney, liver, lung; $n=16 /$ tissue type) were collected from 16 Large White X Minnesota minipig crossbred pigs ( $n=9$ female, $n=8$ male, age range 29-1447 days) at the University of Illinois at Urbana-Champaign. All blood samples were collected in EDTA tubes, aliquoted, and flash frozen in liquid nitrogen within 10 min of collection. Tissue samples were collected and flash frozen within $10 \mathrm{~min}$ of euthanasia. All samples were stored at -80 until processing. Samples were shipped to the University of California, Los Angeles Technology Center for Genomics \&
Bioinformatics for DNA extraction and generation of DNA methylation data.

Human tissue samples

To build the human-pig clock, we analyzed previously generated methylation data from $n=1211$ human tissue samples (adipose, blood, bone marrow, dermis, epidermis, heart, keratinocytes, fibroblasts, kidney, liver, lung, lymph node, muscle, pituitary, skin, spleen) from individuals whose ages ranged from 0 to 93 . The tissue samples came from three sources. Tissue and organ samples from the National NeuroAIDS Tissue Consortium [35]. Blood samples from the Cape Town Adolescent Antiretroviral Cohort study [36]. Skin and other primary cells provided by Kenneth Raj [37]. Ethics approval (IRB\#15-001,454, IRB\#16-000,471, IRB\#18-000,315, IRB\#16-002,028).

DNA methylation data

All methylation data were generated DNA methylation data using the custom mammalian array "HorvathMammalMethylChip40" [38]. Not all CpGs on the array apply to pigs. The particular subset of species for each probe is provided in the chip manifest file at the NCBI Gene Expression Omnibus (GEO) platform (GPL28271). The SeSaMe normalization method was used to define beta values for each probe [39].

\section{Relative age estimation}

To introduce biological meaning into age estimates of pigs and humans that have very different lifespan, as well as to overcome the inevitable skewing due to unequal distribution of data points from pigs and humans across age range, relative age estimation was made using the following formula: Relative age = Age/ maxLifespan, where the maximum lifespan for the two species was chosen from an updated version of the anAge data base [40]. According to the "anAge" data base [40, 41], the maximum lifespan of Sus scrofa is 27 years since one specimen of the riukiuanus subspecies lived for 27 years in captivity [42]. In our models, we chose the more conservative value of 23 years as mentioned in the "Discussion" section. 
Clocks and penalized regression

Details on the clocks (CpGs, genome coordinates) and R software code are provided in the Supplement. Penalized regression models were created with glmnet [43]. We investigated models produced by elastic net" regression (alpha $=0.5$ in glmnet $\mathrm{R}$ function). The optimal penalty parameters in all cases were determined automatically by using a tenfold internal crossvalidation (cv.glmnet) on the training set. By definition, the alpha value for the elastic net regression was set to 0.5 (midpoint between Ridge and Lasso type regression) and was not optimized for model performance. We performed a cross-validation scheme for arriving at unbiased (or at least less biased) estimates of the accuracy of the different DNAm based age estimators. One type consisted of leaving out a single sample (LOOCV) from the regression, predicting an age for that sample, and iterating over all samples. We subset the set of $\mathrm{CpG}$ probes to those that uniquely mapped to a $\mathrm{CpG}$ site in the swine genome. While no transformation was used for the blood clock for pigs, we did use a log linear transformation for the dual species clock of chronological age (Supplement). The accuracy of the resulting clocks was assessed via cross validation estimates of (1) the correlation $R$ between the predicted epigenetic age and the actual (chronological) age of the animal, and (2) the median absolute difference between DNAm age and chronological age (mae).

\section{EWAS and functional enrichment}

Epigenome-wide association studies (EWAS) was performed in each tissue separately (bladder, blood, cerebral cortex, kidney, liver, lung) using the R function "standardScreeningNumericTrait" from the "WGCNA" R package [44]. Next, the results were combined across tissues using Stouffer's meta-analysis method. The functional enrichment analysis was done using the genomic region of enrichment annotation tool [45]. CpGs implicated by our EWAS were filtered for $\mathrm{CpG}$ position information, lifted over to the human genome using UCSC's Liftover tool and fed into the online functional analysis tool GREAT using the default mode, to obtain a list of significantly enriched functions for both positive and negative EWAS hits in the different tissues.
Transcription factor enrichment and chromatin states

The FIMO (Find Individual Motif Occurrences) program scans a set of sequences for matches of known motifs, treating each motif independently [46]. We ran TF motif (FIMO) scans of all probes on the HorvathMammalMethyl40 chip using motif models from TRANSFAC, UniPROBE, Taipale, Taipaledimer, and JASPAR databases. A FIMO scan $p$ value of $1 \mathrm{E}-4$ was chosen as the cutoff (lower FIMO $p$ values reflect a higher probability for the local DNA sequence matching a given TF motif model). This cutoff implies that we would find almost all TF motif matches that could possibly be associated with each site, resulting in an abundance of TF motif matches. We caution the reader that our hypergeometric test enrichment analysis did not adjust for CG content.

\section{Results}

In total, we analyzed 238 tissue samples mainly from blood (Table 1). Blood samples were obtained from three pig lines: a cross between the Large White and Landrace domestic pig breeds, the Wisconsin Miniature Swine minipig, and a cross between the Large White domestic breed (maternal line) and the Minnesota minipig (sire). From the latter pig line (i.e., the domestic minipig cross), methylation profiles were obtained from DNA isolated from bladder, brain (frontal cortex), kidney, liver, and lung tissue. Unsupervised hierarchical clustering revealed that the samples clustered by tissue type (Supplementary Fig. 1). Random forest predictors were fitted to three different outcomes: (1) pig breed (Sus scrofa domesticus versus Sus scrofa minusculus), (2) tissue type, and (3) sex. The three classifiers exhibited perfect accuracy, with respective (out-of-bag) error rates of zero.

Predictive accuracy of the epigenetic clock

To arrive at unbiased estimates of the epigenetic clocks, we applied cross-validation analysis with the training data. For the development of the basic pig clock, this consisted of pig blood, bladder, frontal cortex, kidney, liver, and lung DNA methylation profiles. For the generation of human-pig clocks however, the training data was constituted by human and pig DNA methylation profiles. Cross-validation 
Table 1 Description of the porcine methylation data. Pig lines. Tissue type, $\mathrm{N}=$ total number of samples/arrays. Number of females. Age: mean, minimum and maximum

\begin{tabular}{|c|c|c|c|c|c|c|}
\hline Pig line & Tissue & $\mathrm{N}$ & No. of female & Mean age & Min. age & Max. age \\
\hline Domestic & Blood & 82 & 82 & 2.34 & 0.0301 & 6.26 \\
\hline Wisconsin miniature swine & Blood & 60 & 60 & 0.997 & 0.0219 & 5.15 \\
\hline Domestic/Minnesota mini cross & Blood & 16 & 9 & 1.44 & 0.0795 & 3.96 \\
\hline Domestic/Minnesota mini cross & Bladder & 15 & 9 & 1.54 & 0.0795 & 3.96 \\
\hline Domestic/Minnesota mini cross & Frontal Cortex & 16 & 9 & 1.44 & 0.0795 & 3.96 \\
\hline Domestic/Minnesota mini cross & Kidney & 16 & 9 & 1.44 & 0.0795 & 3.96 \\
\hline Domestic/Minnesota mini cross & Liver & 16 & 10 & 1.61 & 0.0795 & 3.96 \\
\hline Domestic/Minnesota mini cross & Lung & 15 & 8 & 1.54 & 0.0795 & 3.96 \\
\hline
\end{tabular}

analysis reports unbiased estimates of the age correlation R (defined as Pearson correlation between the age estimate (DNAm age) and chronological age) as well as the median absolute error.

From these analyses, we developed four epigenetic clocks for pigs that vary with regard to two features: species and measure of age. The resulting two humanpig clocks mutually differ by way of age measurement. One estimates chronological ages of pigs and humans (in units of years) based on methylation profiles of $638 \mathrm{CpGs}$, while the other employs the methylation profiles of $542 \mathrm{CpGs}$ to estimate relative age, which is the ratio of chronological age of an animal to the maximum lifespan of its species; with resulting values between 0 and 1 . This relative age ratio is highly advantageous because it allows alignment and biologically meaningful comparison between species with very different lifespans such as pig and human, which cannot otherwise be afforded by direct comparison of their chronological ages.

As indicated by its name, the pure pig clock, constituted by $120 \mathrm{CpGs}$, is highly accurate in age estimation in all porcine tissues $(R=0.97$ and median error 0.22 years, Fig. 1A). The pan-tissue clocks exhibit high age correlations in individual porcine tissues ( $R>0.90$, Supplementary Fig. 1$)$. The humanpig clock for chronological age is highly accurate when DNA methylation profiles of both species are analyzed together $(R=0.98$, Fig. $1 \mathrm{~B})$, and remains so when restricted to pig tissue samples $(R=0.97$, Fig. 1C). Similarly, the human-pig clock for relative age exhibits high correlation regardless of whether the analysis is applied to samples from both species ( $R=0.98$, Fig. 1D) or only to pig samples $(R=0.96$, Fig. 1E). The use of relative age circumvents the clustering of data points of pigs and humans to opposite parts of the curve, which is evident in Fig. 1C. These highly accurate array of porcine clocks are readily useable with immediate effect in porcine models of diseases and conditions, and the humanpig clock of relative age is particularly exciting as it allows comparison between human and pigs based on their relative positions within the lifespans of both species.

\section{EWAS of chronological age}

Although several hundred CpGs were used to construct the epigenetic clocks described above, these were merely a subset of all $\mathrm{CpGs}$, which changed with advancing age. There are many more age-associated CpGs that are not used for the purpose of estimating porcine age, but are nevertheless very important when we seek to identify CpGs with methylation levels that are associated with age through EWAS. In total, 34,540 probes from the HorvathMammalMethylChip40 are aligned to loci that are proximal to 5,209 genes in the Sus_scrofa.Sscrofal1.1.100 genome assembly. Due to the high inter-species conservation of the probes on the array, findings from the pig methylation data can probably be extrapolated to humans and other mammalian species. EWAS of chronological age revealed clear tissue-specific DNAm change in pigs (Fig. 2A). Age-associated CpGs in one tissue tend to be poorly conserved in another tissue (Supplementary Fig. 3). However, the poor conservation and differences in $p$ value ranges in our analyzed tissue types may reflect the limited sample size in nonblood tissues. 
Leave-One-Out Analysis of All Final Epigenetic Clocks
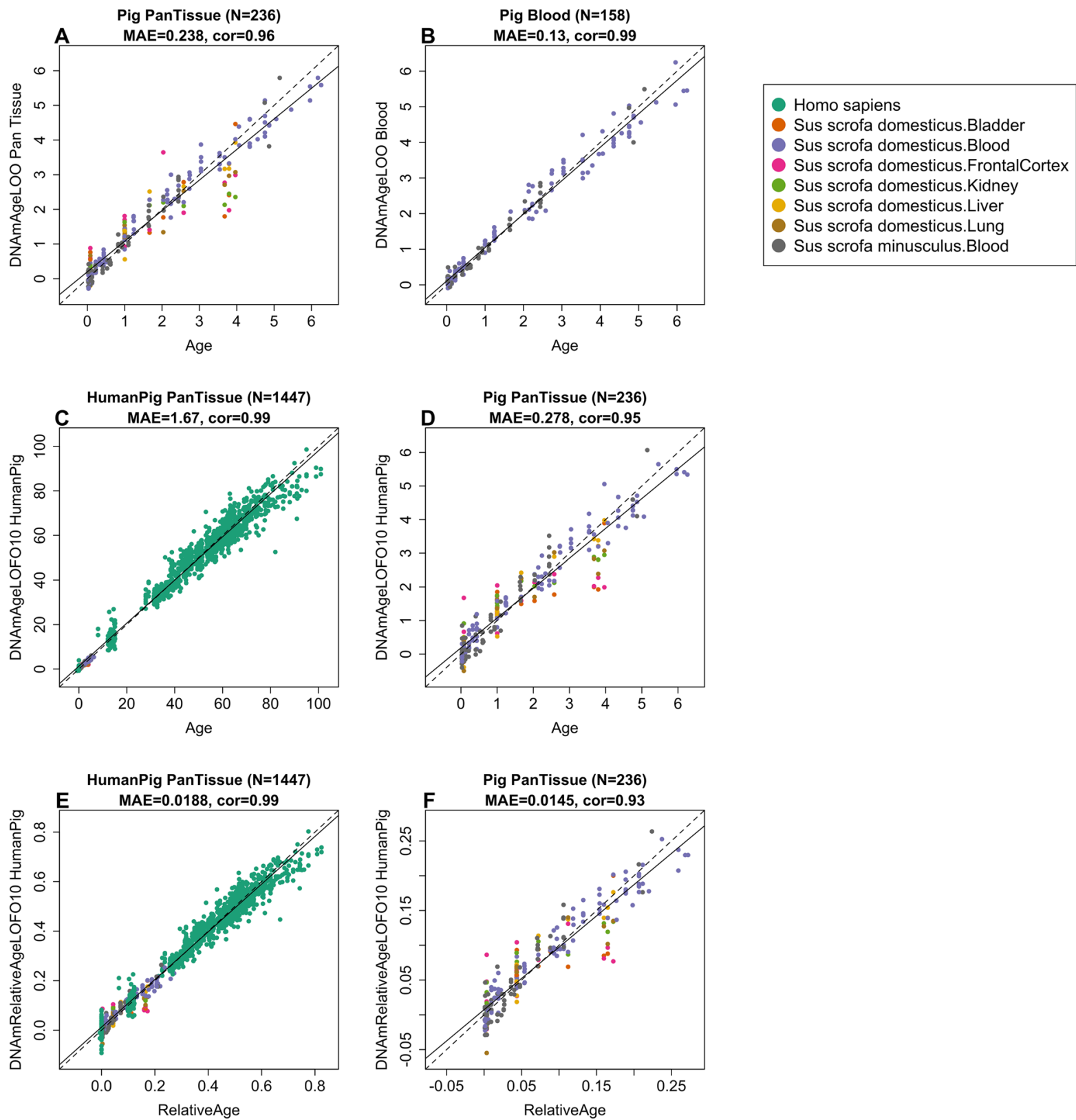

Fig. 1 Cross-validation study of epigenetic clocks for pigs. We developed 4 epigenetic clocks for pigs: A Pan-tissue clock, B blood clock, and human-pig clock for chronological age applied to $\mathbf{C}$ both species and $\mathbf{D}$ pigs only. Human-pig clock for relative age applied to $\mathbf{E}$ both species and $\mathbf{F}$ pigs only. Leave-one-sample-out (LOO) estimate ( $y$-axis, in units of years) versus chronological age or relative age ( $x$-axis). Relative age is defined as chronological age divided by the maximum age of the respective species. The linear regression of epigenetic age is indicated by a solid line while the diagonal line $(y=x)$ is depicted by a dashed line
A nominal $p$ value $<10^{-4}$ was set as the cutoff for significance. The top age-associated $\mathrm{CpGs}$ and their proximal genes for the individual tissues are as follows: bladder, ANO4 exon $(z=5.8)$; blood, EN1 
promoter $(z=26)$; frontal cortex, $F G F 9$ exon $(z=5.6)$; kidney, TNRC6A exon $(z=-7.5)$; liver, NRSA1 exon $(z=8.6)$; and lung, UNC79 5'UTR $(z=8.1)$. Despite poor conservation across tissues, there are nevertheless age-related $\mathrm{CpGs}$ that are common to all tissues, and these were identified through meta-analysis of these six tissue samples to be hypermethylation in EN1 promoter $(z=18), H C N 1$ exon $(z=17), U N C 79$ 5'UTR $(z=17)$, LHFPL4 exon $(z=17)$, and NR2F2 exon $(z=17)$. The upset plot analysis identified several CpGs with conserved DNAm aging in at least four pig tissues (Fig. 2B). The most conserved DNAm aging pattern was hypermethylation of the SP8 promoter in all tissues, with the exception of the brain. Genes whose expressions are regulated by SP family transcriptional factors are essential for proper limb development. Aging-associated CpGs in different tissues were distributed in genic and intergenic regions that are defined relative to transcriptional start sites (Fig. 2C). There is little enrichment of agerelated CpGs in any genetic features across all tissues, with the exception of consistent hypermethylation in promoters and 5'UTRs. This result is consistent with a higher positive association of $\mathrm{CpG}$ island methylation with age than non-island $\mathrm{CpGs}$ in all tissues (Fig. 2D). These features suggest that a substantial amount of age-associated CpGs are likely to impact gene expression.

To specifically explore the potential impact of agerelated porcine $\mathrm{CpG}$ methylation on gene expression, we analyzed putative transcription factors binding sites for such methylation. From these we identified 20 transcription factor binding motifs that exhibit age-related methylation changes (Fig. 3A). The corresponding transcription factors control the expression of genes which are involved in many different cellular activities. For example, hypomethylation in the SP1 motif in blood and cortex indicates greater access of the SP1 protein to some of its binding sites with increasing age. However, the outcome of this is difficult to predict as SP1 activates the transcription of many genes that are involved in diverse cellular processes ranging from cell growth, apoptosis, the immune response and chromatin remodeling. The challenge in predicting downstream events from single transcription motifs can be partly addressed by collective analysis of multiple transcription factor motifs. Such an analysis identified age-associated methylation changes for SMAD3, SP1, SP3, and
E2F1 transcription factor binding motifs, which are implicated in telomerase regulation $(p=6 \mathrm{E}-7)$. We briefly mention that the effects of telomere length and telomerase activity in porcine tissues appears to be comparable with that of humans [47].

While identification of genes proximal to age-associated CpGs, as performed above, are useful, the likelihood of their potential effects on cells is difficult to gauge. This can be partly addressed by carrying out analysis to identify enrichment of implicated genes in specific pathways, pathologies and biological processes (Fig. 3). This analysis highlighted the following features impacted by age-related $\mathrm{CpG}$ methylation changes in porcine cells: organism development, the nervous system and metabolism; all of which have also been found to be implicated in epigenetic aging of humans and other species (Fig. 3B). Furthermore, genes proximal to hypermethylated CpGs are associated with H3K27Me3 marks and are often polycomb protein EED targets in porcine tissues. EED is a member of the multimeric Polycomb family protein complex that maintains the transcriptional repressive states of genes. These proteins also regulate H3K27Me3 marks, DNA damage, and senescence states of the cells during aging [48].

Studying differences across pig lines

With the DNA methylation datasets we have accrued from the different porcine breeds, we are in a position to identify $\mathrm{CpGs}$ that differ between domestic and minipig breeds. We compared DNA methylation profiles from blood of domestic pigs with those from Wisconsin Miniature Swine ${ }^{\mathrm{TM}}$.

We found the mean methylation across CpGs located in $\mathrm{CpG}$ islands is higher in minipigs than in the other two pig lines (Supplementary Fig. 4). Similarly, the average rate of change in methylation across island CpGs is increased in minipigs (Supplementary Fig. 4).

We analyzed individual $\mathrm{CpGs}$ using two different multivariate models. In the first, the DNAm levels of a given $\mathrm{CpG}$ were regressed on age and breed (minipig versus reset) to identify CpGs that are associated with aging in both breeds (age main effect). This model was also used to identify CpGs with significantly different basal methylation levels between breeds (Minipig main effect). The second model identified age-related CpGs with different 
EWAS of chronological age

A
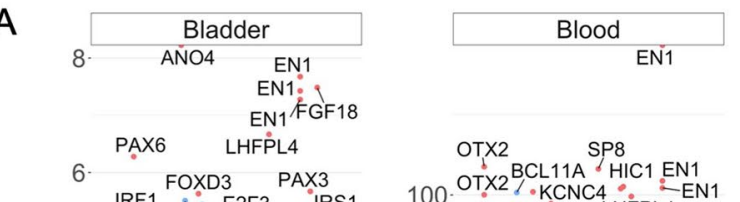
\begin{tabular}{|l|l|}
\hline \multicolumn{2}{|c|}{ Frontal Cortex } \\
OSR2 FGF9 HOXA3 \\
HOXC5
\end{tabular}

ONECUT1 1 TFAP2A HOXA3

6. GPR61 $\mathrm{BMF}_{8}$ \& RTN4RL1

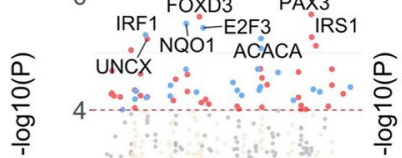

2.

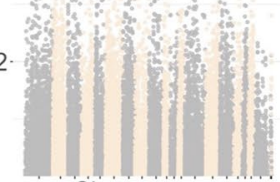

Chromosome

Liver
16. NR5A1PAX9.UNC79
TFAP2D ${ }^{\circ}$ FOXG1 GPC6
PAX6 UNC79 SATB1

PAX6 UNC79 SATB1

UNGX ANKS1B

12. $\mathrm{HOXC11} \cdot \cdots$
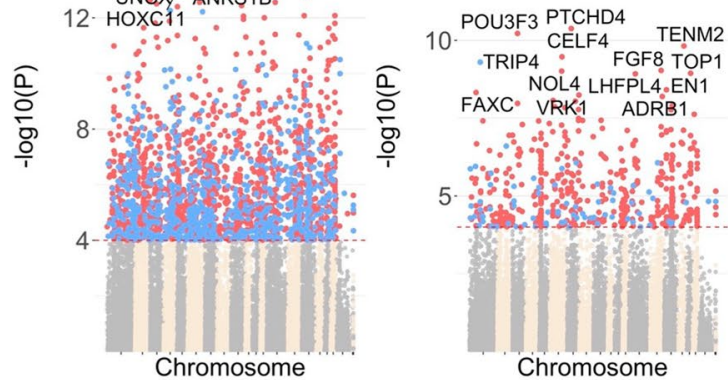

50 .

OTP DMRK

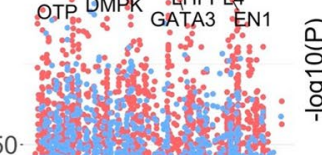

$\because \therefore$

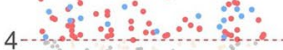

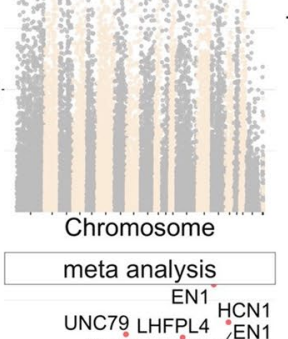

UNC79 LHFPL4 HCN1

60. POU3F3 ${ }^{\mathrm{NR} 2 \mathrm{~F} 2}$ LHFPL44

PHACگIRR $144^{\circ} 8^{\circ}$

OTP: PTCHDA RGS7BP

$\because \because 8 \%$ :

มี:ㅇำ

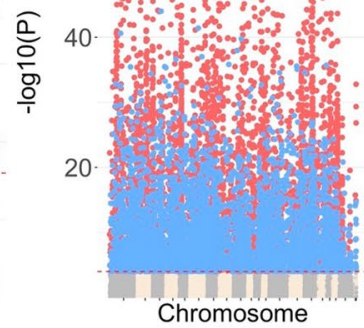

\begin{tabular}{|r|}
\hline Kidney \\
\hline
\end{tabular}

SYNGAP1 RAB11FIP4

10.0. PABPC4

MAPKAP1 RSBN1 1 RXW11

PAX6 VRK2 ATXN7

등 7.5. $\begin{aligned} & \text { LIN28B ZFHX4 ATXN7 } \\ & \text { SERED2 RNF144A }\end{aligned}$

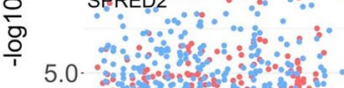

2.5

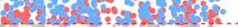

Chromosome

Direction of association

Hypermethylated

Hypomethylated
B

Upset plot of the significant $\mathrm{CpGs}$

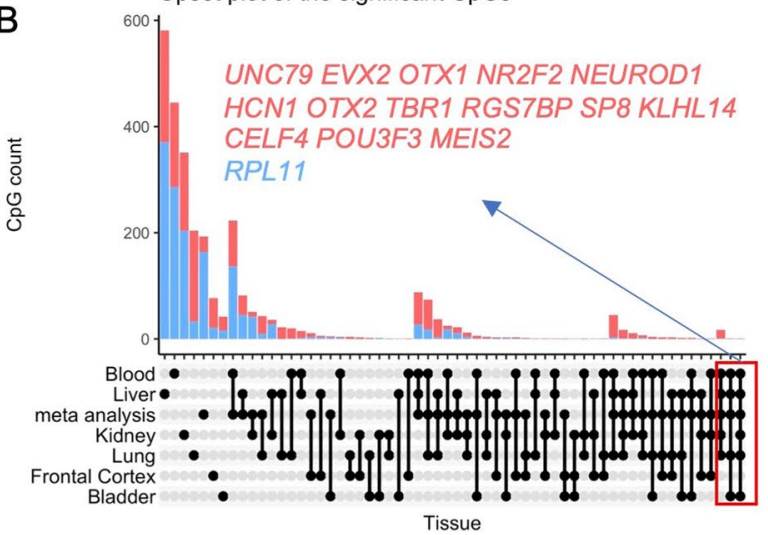

D
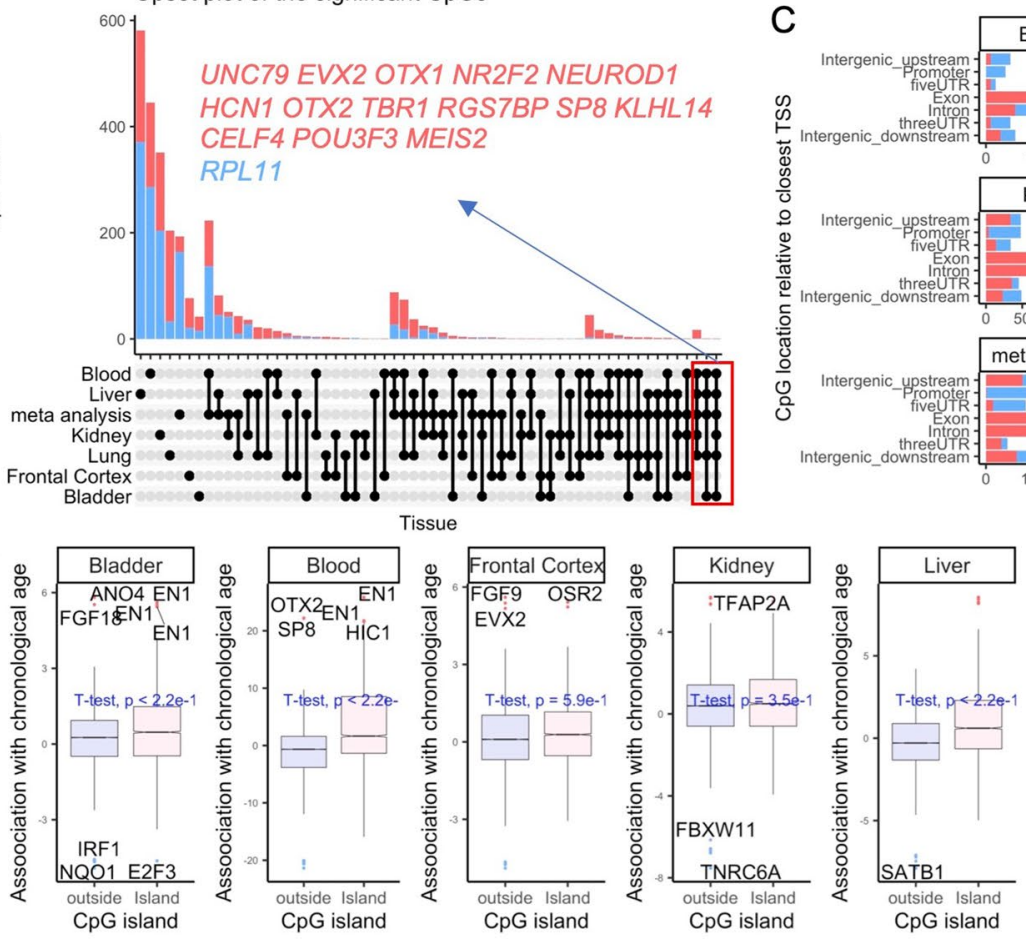
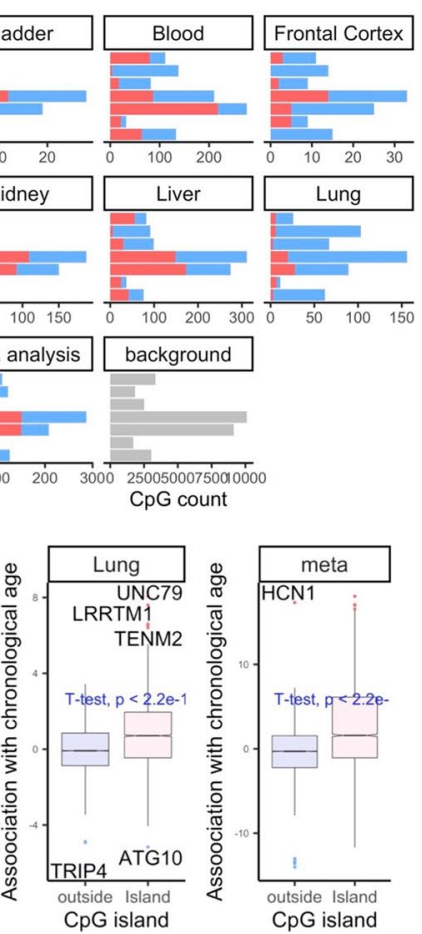
4Fig. 2 Epigenome-wide association (EWAS) of chronological age in porcine tissues. EWAS of age in bladder, blood, frontal cortex, kidney, liver, lung, and tissue meta-analysis of pigs (Sus scrofa). A Manhattan plots of the EWAS of chronological age. The coordinates are estimated based on the alignment of the Mammalian array probes to the Sscrofa11.1.100 genome assembly. The direction of associations with $p<10^{-4}$ (red dotted line) is highlighted by red (hypermethylated) and blue (hypomethylated) colors. The top $30 \mathrm{CpGs}$ were labeled by their neighboring genes. B Upset plot representing the overlap of aging-associated CpGs based on meta-analysis or individual tissues. Neighboring genes of the overlapping CpGs are labeled in the figure. C Location of top CpGs in each tissue relative to the closest transcriptional start site. Top $\mathrm{CpGs}$ were selected at $p<10^{-4}$ and further filtering based on $\mathrm{z}$ score of association with chronological age for up to 500 in a positive or negative direction. The number of selected CpGs: bladder, 73; blood, 1000; frontal cortex, 123; kidney, 574; liver, 1000; lung, 528; and tissue meta-analysis, 1000 . The grey color in the last panel represents the location of the 34,540 mammalian BeadChip array probes mapped to Sscrofa11.1.100 genome. D Boxplot of DNAm aging for CpGs located within or outside $\mathrm{CpG}$ islands in porcine tissues. Labels indicate neighboring genes of the top $10 \mathrm{CpGs}$ in each analysis

rates of methylation changes between these two pig breeds independent of the direction of change. At a genome-wide significance set at $\mathrm{p}<1 \mathrm{e}-8 ; 10,167$ age-associated $\mathrm{CpGs}$ were shared between both pig breeds, while $825 \mathrm{CpGs}$ had different baseline methylation levels, and the rate of methylation change of 32 age-associated $\mathrm{CpGs}$ were significantly different between the two (Fig. 4A). Thus, while age has the largest impact on methylation of these CpGs, there is an inherent species-specific difference in basal methylation levels of a substantial number of CpGs, which is expected given the overt differences between the breeds. The top CpGs with divergent rate of methylation change between the breeds are proximal to the MGST1 exon, SON 3'UTR, and TFAP2B exon (Fig. 4A-C). CpGs with different basal methylation levels between the two breeds were located within the $O S B P$ exon, ENCl intron, and CTNNBL1 upstream regions (Fig. 4A). In total, eight categories of CpGs can be defined based on the direction of their methylation change with age in the two pig breeds. While methylation of most age-associated $\mathrm{CpGs}$ was altered in similar directions in both breeds (hypo or hyper in both axes), some were clearly in opposite directions (hyper in one axis and hypo in the other). The LMNA intron is an example region that displayed extreme divergence. While its intron was hypomethylated with age in the domestic pig, it was hypermethylated in minipigs (Fig. 4C). An enrichment analysis of agerelated CpGs that are exclusive to either breed implicated pathways involved in development, survival, cancer, and growth (Fig. 4D).

Our analysis of differentially methylated CpGs between domestic and minipigs implicated genes that regulate weight. This particularly interesting finding prompted us to query whether any of these identified genes are associated with weight differences in humans. To this end, we overlapped the EWAS genes with a large GWAS meta-analysis of body-mass index (BMI) in humans, which included 681,275 participants in UK Biobank and GIANT BMI $_{\text {I }}$ consortium [49]. Strikingly, several of the EWASidentified genes had genetic variants that are associated with increased BMI in humans (Fig. 5). The top genes include ADCY3, TFAP2B, SKOR1, and GPR61, which had numerous SNPs in different gene regions associated with human BMI. Thus, our EWAS analysis of pig breeds of overtly different sizes implicates genes that were implicated by a published GWAS of human BMI.

\section{Discussion}

We have previously developed several human epigenetic clocks from DNA methylation profiles that were derived from various versions of human Illumina DNA methylation arrays. As these arrays are specific to the human genome, a critical step toward crossing the species barrier was the development and use of a mammalian DNA methylation array that profiles 36,000 CpGs with flanking DNA sequence that are highly conserved across numerous mammalian species. The employment of this array to profile 238 porcine tissues from 3 pig lines represents the most comprehensive epigenetic dataset of domestic pigs thus far. These data allowed us to construct four highly accurate DNA methylation-based age estimators for three pig lines. Two of these clocks apply only to pigs: the pan-tissue clock which was trained on methylation profiles of six tissues is expected to apply to most pig tissues. The blood clock on the other hand, was trained using only blood DNA methylation data. These two highly accurate porcine clocks are readily and easily included in porcine-based models of diseases and health conditions. This will encourage investigations into the relationship between age and 
A

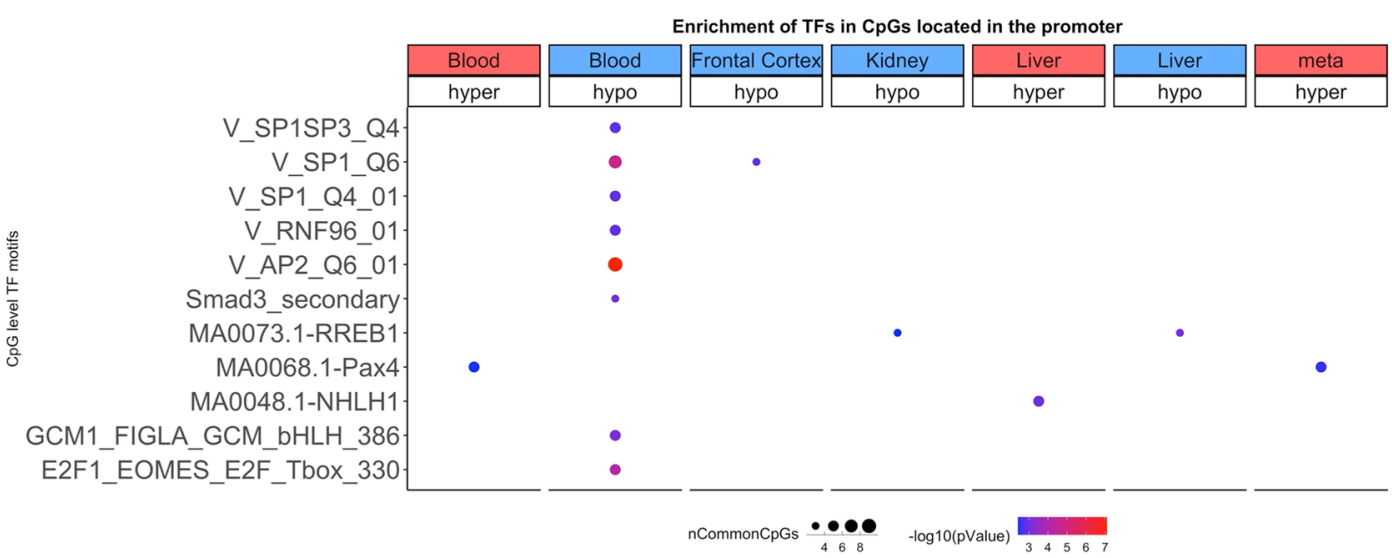

B

\begin{tabular}{|c|c|c|c|c|}
\hline & \multicolumn{4}{|c|}{ Enrichment for GO,phenotypes, upsptream regulators } \\
\hline & Blood & Liver & Lung & meta \\
\hline & hyper & hyper & hyper & hyper \\
\hline BENPORATH_ES_WITH_H3K27ME3. & $\cdot$ & . &. & $=$ \\
\hline BENPORATH_EED_TARGETS & $\cdot$ & - & - & - \\
\hline$\overline{\mathrm{DNA}}$ binding & - & - & - & - \\
\hline single-multicellular organism process & - & $\bullet$ & $\bullet$ & - \\
\hline multicellular organismal process & - & - & - & - \\
\hline multicellular organismal development & - & - & - & - \\
\hline developmental process & - & - & $\bullet$ & - \\
\hline anatomical structure development & - & $\bullet$ & $\bullet$ & - \\
\hline NA binding transcription factor activity & - & - & - & - \\
\hline acid binding transcription factor activity & - & - & • & - \\
\hline nervous system phenotype & $\Delta$ & $\Delta$ & $\Delta$ & $\Delta$ \\
\hline sequence-specific DNA binding & $\bullet$ & - & - & - \\
\hline abnormal nervous system morphology & $\Delta$ & $\Delta$ & $\Delta$ & $\Delta$ \\
\hline decreased brain size & 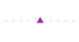 & & & 1 \\
\hline BENPORATH_SUZ12_TARGETS. & - & - & - & - \\
\hline ity during fetal growth through weaning & $\Delta$ & $\Delta$ & $\Delta$ & $\Delta$ \\
\hline BENPORATH_PRC2_TARGETS & $\cdot$ & - & - & - \\
\hline al somatic nervous system morphology & $\Delta$ & $\Delta$ & $\Delta$ & $\Delta$ \\
\hline organ development. & - & - & - & - \\
\hline V\$CEBPA 01 & . & & & . \\
\hline abnormal neuron morphology. & $\Delta$ & $\Delta$ & $\Delta$ & $\Delta$ \\
\hline regionalization & • & • & • & . \\
\hline abnormal olfactory lobe morphology &. & . & & 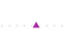 \\
\hline KELSEN_IPS_WITH_HCP_H3K27ME3. & $\cdot$ & - & & $\cdot$ \\
\hline$-{ }_{-}-$periñatal lethality & $\Delta$ & $\Delta$ & $\Delta$ & $\Delta$ \\
\hline small olfactory bulb & 1 & & & 1 \\
\hline complete perinatal lethality & $\Delta$ & . & & 4 \\
\hline CTTTAAR_UNKNOWN & $\cdot$ & & & $\cdot$ \\
\hline abnormal olfactory bulb $\overline{\text { development }}$ & 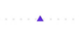 & & & 1 \\
\hline abnormal neuron proliferation & $\Delta$ & & & $\Delta$ \\
\hline organ morphogenesis & $\bullet$ & & & - \\
\hline dorsal/ventral pattern formation & - & - & & - \\
\hline V\$GFI1_01. & $\cdot$ & & $\cdot$ & $\cdot$ \\
\hline V\$NKX22-01 & & & & $\cdot$ \\
\hline truncation of digits & . & & & 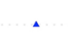 \\
\hline ectopic dopaminergic neuron- &. & & & s. \\
\hline absent cerebellar lobules. &. & & & . \\
\hline abnormal digit pigmentation & 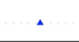 & & & 4 \\
\hline
\end{tabular}


4Fig. 3 Enrichment analysis of the top DNAm aging marks in porcine tissues. A Transcriptional motif enrichment for the top CpGs in the promoter (and 5'UTR) of the neighboring genes. The motifs were scanned using the FIMO for all the probes, and the enrichment was tested using a hypergeometric test. B) Gene set enrichment analysis of proximate genes with DNAm aging in porcine tissues. The analysis was done using the genomic region of enrichment annotation tool [45]. The gene level enrichment was done using GREAT analysis [45] with the human $\mathrm{Hg} 19$ background. The background probes were limited to 23,087 probes that were mapped to the same gene in the pig genome. The top three enriched datasets from each category (canonical pathways, diseases, gene ontology, human and mouse phenotypes, and upstream regulators) were selected and further filtered for significance at $p<10^{-8}$

diseases, and also uncover the effects of environment, living condition, food, medicine, and treatment on the rate of porcine epigenetic aging. While toxicity, mutagenicity and carcinogenicity are considered healthimpacting effects that draw the attention of health experts, age-accelerants have yet to be appreciated as being potentially as important, or perhaps even more so, as age is the biggest risk factor for almost all diseases, and accelerated epigenetic aging is associated with a whole host of pathologies and health conditions. While mice can be used to detect potential ageaccelerating agents, this route is evidently limited due to the inherent differences between humans and mice, such as food, sleep-wake cycle, metabolism, physiology, endocrinology, and disease susceptibility. The better compatibility between pigs and humans are elaborated above, and the availability of these porcine epigenetic clocks further consolidates the greater relevance of employing porcine models for human health conditions. This advantage is compounded by the successful generation of human-pig clocks that apply to both species. It is essential to appreciate the profound significance of these dual species clocks. With a single mathematical formula, the age of humans and pigs can be estimated based solely on methylation levels of a few hundred cytosines.

Some of the loci that harbor age-associated CpGs in pigs and humans (as well as other mammalian species), are bivalent chromatin domains and targets of the polycomb repressive complex 2 (PRC2). This is of significance because these regions are primarily located upstream of Hox genes which specify development of various parts of the body of organisms ranging from worms, flies, mice and humans. In other words, these are some of the oldest genetic elements of life. In this study the highest scoring porcine blood $\mathrm{CpG}$ is one that resides in the promoter of the engrailed gene (EN1), which is a member of the Hox gene family. EN1 also scored the highest in metaanalyses of all the tissues. It is as yet unclear how methylation of these loci are involved with aging, but their increased methylation with age hints at the possible role of cellular identity and differentiation. This is no doubt an exciting avenue of exploration in which these epigenetic clocks will be essential.

The considerable similarities between humans and pigs allows for the testing of age-mitigation interventions as well as factors that impact longevity in pigs, which have a shorter lifespan and are much more amenable subjects for controlled trials. To accurately translate age-related findings from pigs to humans however requires a correct and accurate measure of age-equivalence. We fulfilled this need through a two-step process. The first, which we described above is the generation of dual-species clocks (human-pig), one of which is as accurate in estimating pig age as it is for human age; in chronological units of years. The second is the expression of pig and human ages in respect to the maximum recorded ages of their respective species (species lifespan), i.e. 23 years for pigs and 122 years for humans. It is difficult to find reliable lifespan data for domestic pigs.

According to the "anAge" database [40, 41], the maximum lifespan of Sus scrofa is 27 years since one specimen of the riukiuanus subspecies lived for 27 years in captivity [42]. In our models, we chose the more conservative value of 23 years after consulting with experts. We are not sure whether minipigs live longer lives than regular sized domestic pigs. If in fact pigs behave the same as dogs (and several other species), one would expect domestic pigs to live considerably less long than minipigs. Minipigs are expected to live between 12 and 18 years but precise estimates of maximum lifespan appear to be unknown $[50,51]$. In our own database, we assigned the Wisconsin Miniature Swine ${ }^{\mathrm{TM}}$ the Latin name "Sus scrofa minusculus" with an estimated maximum lifespan of 23 years. Domestic pigs experience a remarkably fast growth period which might influence their lifespan, their aging process, and mean methylation levels. This might explain why the mean methylation across CpGs located in $\mathrm{CpG}$ islands is higher in minipigs than in the other two pig lines (Supplementary Fig. 4). 


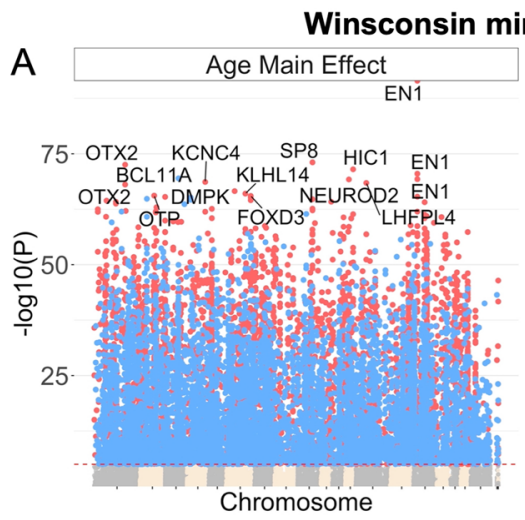

B Age interaction with mini pig
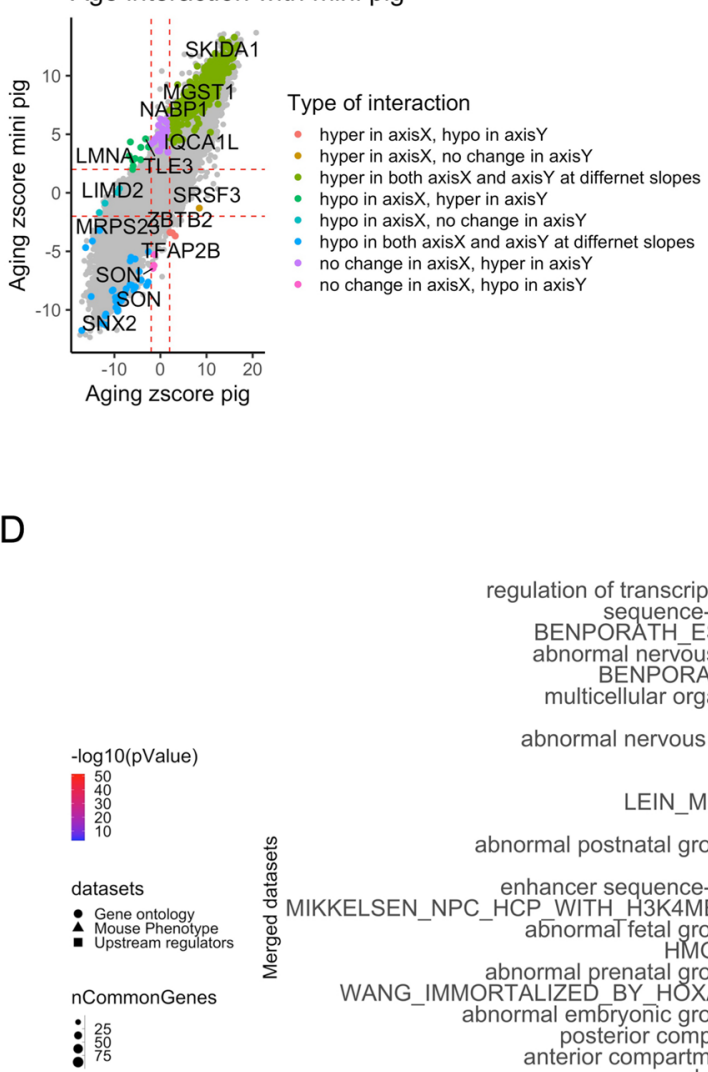
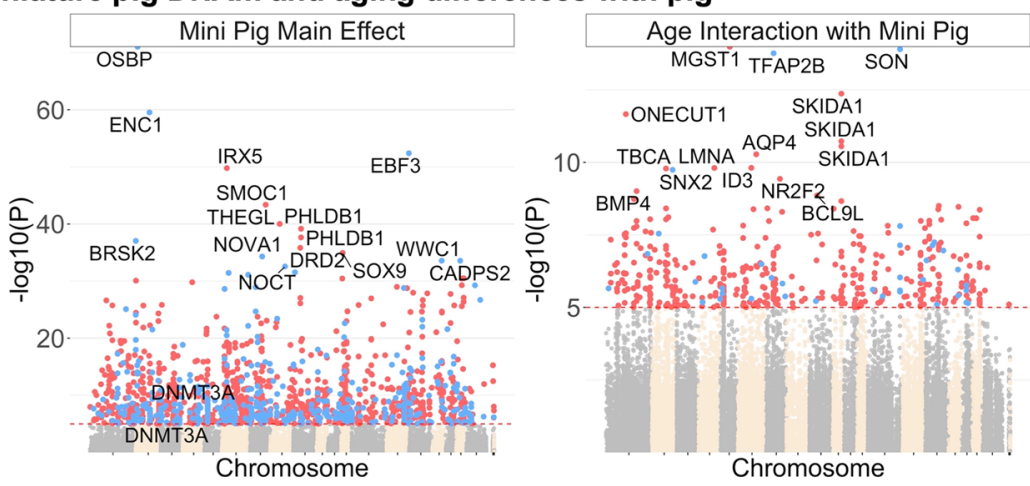

C

species at pig at mini pig
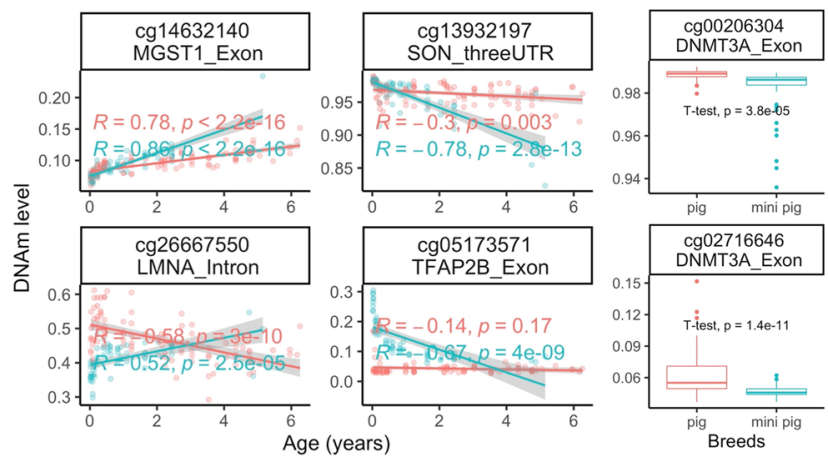

Interaction of age and mini pig

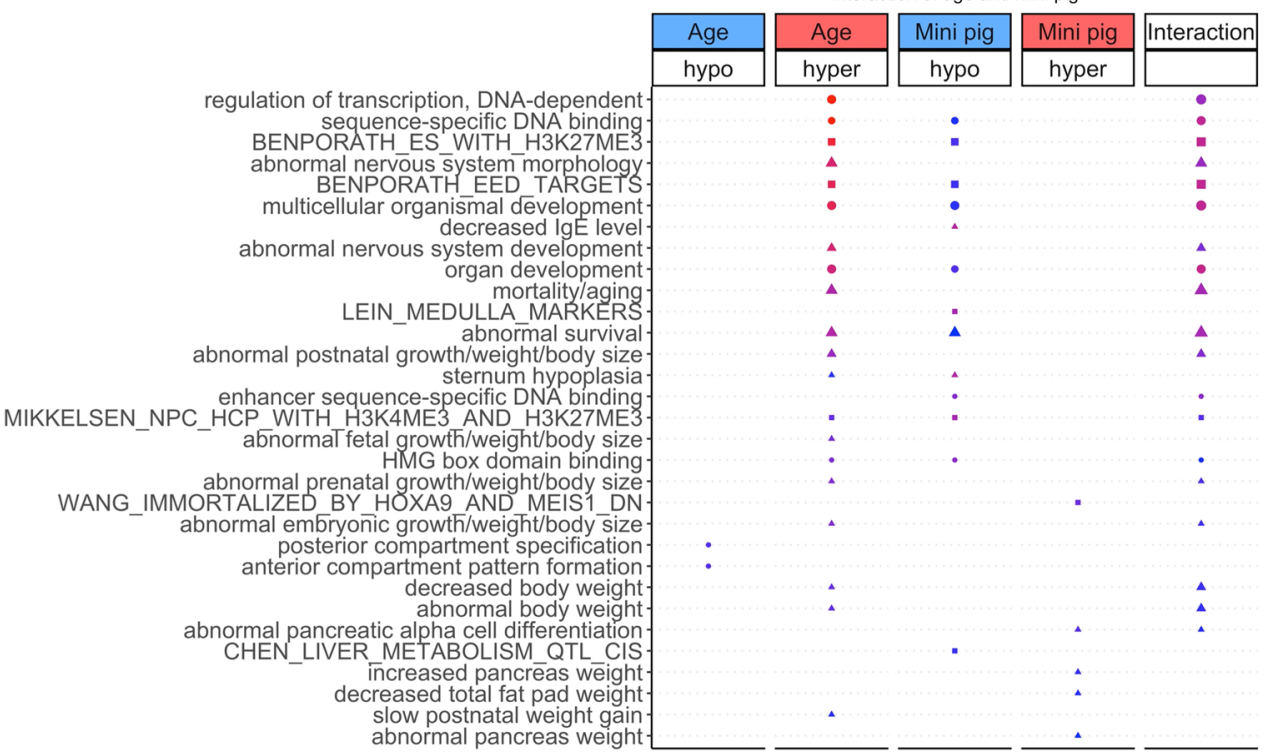

Our study is limited in that all animals were younger than 6.3 years old while the maximum observed lifespan of domestic pigs (Sus scrofa domesticus) appears to be 23. We hypothesize that the lack of training data on older animals resulted in porcine clocks that will under-estimate the ages of older animals. However, we expect that the correlation between age and its DNA methylation based estimate will continue to be high in older animals. Further, we hypothesize that $\mathrm{CpGs}$ that correlate with age in 
4Fig. 4 EWAS of multivariate regression models for pig breed and age. Aging differences between domestic and minipig breeds. The figure visualizes the results of 2 different linear regression models which used individual $\mathrm{CpGs}$ as dependent variable. The 2 linear models differ in terms of the underlying covariates: two covariates (age and pig breed) or three covariates (age, pig breed, and interaction effect). A Manhattan plots of DNAm aging loci that are shared between minipigs and domestic pigs (aging main effect), basal breed differences in DNAm levels (minipig main effect), and the interaction of breed and aging, which represent the loci with a divergent DNAm aging pattern between minipigs and domestic pigs. The analysis is done by multivariate regression models with or without (to estimate the main effect) interaction term for age and breeds. For breeds, the domestic pig is the reference variable to estimate the direction of change. Sample sizes: domestic pigs, 98; minipigs, 60. The red line in the Manhattan plot indicates $\mathrm{p}<1 \mathrm{E}-35$. B Scatter plots of DNAm aging between minipigs and domestic pigs. The highlighted CpGs are the loci with significant DNAm aging interaction between breeds at a 5\% FDR rate. In total, eight categories of interaction were defined based on the aging Z-statistic of each breed. C Age versus methylation levels for select CpGs with significant interaction terms between breed and age. D Enrichment analysis of the genes proximate to $\mathrm{CpGs}$ related to age (shared between breeds), minipig, and age/minipig interaction. The gene-level enrichment was done using GREAT analysis [45] and human $\mathrm{Hg} 19$ background. The background probes were limited to 23,087 probes that were mapped to the same gene in the pig genome. The top CpGs were selected at a $p<1 \mathrm{E}-5$ and based on beta values of association for up to 500 in a positive or negative sign younger animals also correlate with age in older animals. These hypotheses derive from our studies of humans and many other species where these hypotheses have been validated [31, 52].

The mathematical operation of generating a ratio eliminates chronological units of time and produces a value that indicates the age of the organism in respect to the maximum age of its own species. This allows a meaningful and realistic cross-species comparison of biological age. For example, the biological fitness of a 20 -year-old pig, which is very old, is not equivalent to that of a 20 year-old human, who is young. However, a pig with a relative epigenetic age of 0.5 is more comparable to a human of similar relative epigenetic age. Collectively, the ability to use a single mathematical formula to measure epigenetic age of different species and the replacement of chronological units of time with proportion of lifespan, are two significant innovations that will propel cross-species research as well as cross-species benefits.

In addition to age-related epigenetic changes, we also compared DNA methylation profiles between domestic and minipigs. CpGs with substantially

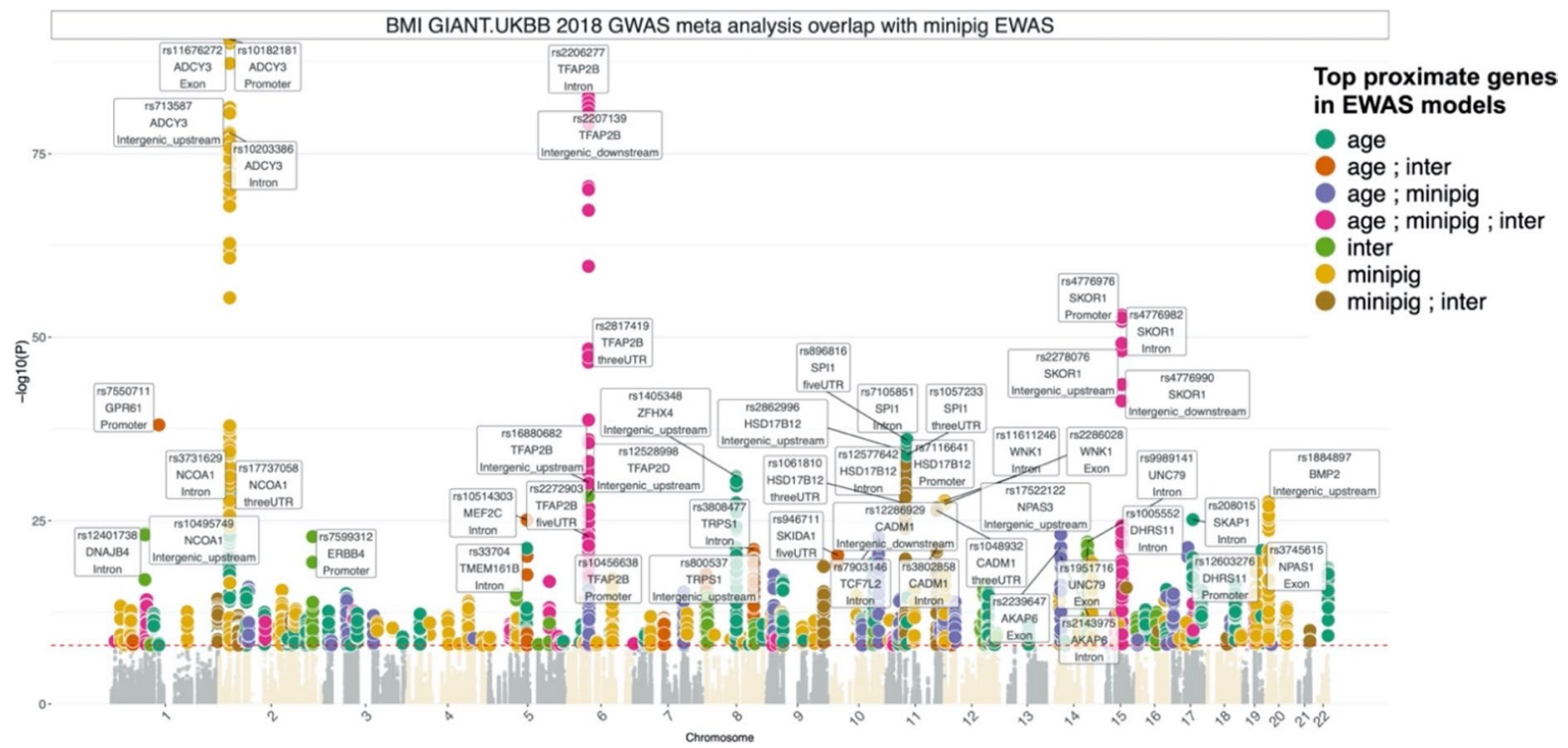

Fig. 5 Overlapping EWAS results in pigs with GWAS results in humans. EWAS of aging, minipig, and aging/pig breed interaction identifies genes with genetic variants associated with human body mass index. The GWAS is based on summary statistics of BMI meta-analysis of 681,275 participants in the UK Biobank and GIANT ${ }_{\mathrm{BMI}}$ consortium [49]. The coloring is based on the genes identified by one or multiple EWAS of age, minipig, and age/minipig interaction (inter). The labels indicate the top SNP from each of the top 30 gene regions 
different basal methylation levels between the two breeds were identified proximal to the $O S B P$ gene. This is of great significance, as OSBP is a transport protein that translocates sterols from lysosomes into the nucleus where the sterol represses the expression of the $L D L$ receptor gene, $H M G-C o A$ reductase gene and $H M G$ synthase gene. The reduction of LDL receptor increases the risk of atherosclerosis and cardiovascular disease. Indeed, mice with ablated $L D L$ receptor genes develop plaques in their aortas, while wild-type mice are free of such plaques. Compounding the effects on LDL receptor levels is the effect on expression of HMG-CoA and HMG synthase, which are members of the intracellular pathway that synthesize cholesterol. These features are of particular interest given the fact that while domestic pigs are refractive to atherosclerotic plaque development, minipigs are susceptible and hence used as models for cardiovascular disease. Another feature of interest is the identification of differentially methylated CpGs associated with genes involved in the development of obesity. The matching of EWAS targets with a large GWAS meta-analysis of body mass index (BMI) in humans led to the identification of overlapping genes included TFAP $2 B$, which influences the effect of dietary fat on weight [53], GPR61, which is involved in the regulation of food intake and body weight $[54,55]$, and $A D C Y 3$ and SKORI, both of which are associated with obesity and BMI [56-59]. Collectively, these associations points to the contribution of epigenetic control and influence on weight gain and obesity, in addition to highlighting the translational relevance of porcine models for cardiovascular disease and obesity-related research. It is interesting to note that the Dnmt3a locus is also differentially methylated between these two breeds. It is tempting to speculate that this may be an upstream event that impacts on the downstream methylation differences described above.

As porcine biomedical models for a wide range of age-related disorders are currently in use or being developed, including Alzheimer's disease [60], cardiovascular disease [61], diabetes [22], and cancer models $[21,23,24]$, the availability of these epigenetic clocks will extend the use of porcine models for aging, and possibly obesity studies.

To rigorously evaluate anti-aging treatment effects, we recommend to randomize treatment assignment and to collect several tissue types, e.g., skin samples (ear punches) and blood samples since treatment effects can be tissue/cell type specific as is the case in menopausal hormone therapy [62]. To average out technical variability in DNAm age estimates, we recommend to collect at least two tissue samples before treatment and at least two samples after treatment per animal.

Acknowledgements We would like to thank Dr. Ana Cecilia Escobar Lopez, Jamie Reichert, Jennifer Frank, Justin Hickman, Keri Graff, and Nathan Chesmore for their technical assistance during the sampling of the Wisconsin Miniature Swine.

Author contribution SH conceived of the study and wrote the article. The remaining authors helped with the statistical analysis or the data generation. All authors reviewed and edited the article.

Funding This work was supported by the Paul G. Allen Frontiers Group (SH).

\section{Declarations}

Conflict of interest SH is a founder of the non-profit Epigenetic Clock Development Foundation which plans to license several of his patents from his employer UC Regents. The other authors declare no conflicts of interest.

Open Access This article is licensed under a Creative Commons Attribution 4.0 International License, which permits use, sharing, adaptation, distribution and reproduction in any medium or format, as long as you give appropriate credit to the original author(s) and the source, provide a link to the Creative Commons licence, and indicate if changes were made. The images or other third party material in this article are included in the article's Creative Commons licence, unless indicated otherwise in a credit line to the material. If material is not included in the article's Creative Commons licence and your intended use is not permitted by statutory regulation or exceeds the permitted use, you will need to obtain permission directly from the copyright holder. To view a copy of this licence, visit http://creativecommons.org/licenses/by/4.0/.

\section{References}

1. Kumar S, Hedges SB. A molecular timescale for vertebrate evolution. Nature. 1998;392:917-920. [Online]. Available: https://doi.org/10.1038/31927.

2. Meredith RW, et al. Impacts of the Cretaceous Terrestrial Revolution and KPg extinction on mammal diversification, (in eng). Science. 2011;334(6055):521-4. https://doi. org/10.1126/science.1211028.

3. Caliebe A, Nebel A, Makarewicz C, Krawczak M, KrauseKyora B. Insights into early pig domestication provided by 
ancient DNA analysis, (in eng). Sci Rep. 2017;7:44550. https://doi.org/10.1038/srep44550.

4. Groenen MA, et al. Analyses of pig genomes provide insight into porcine demography and evolution, (in eng). Nature. 2012;491(7424):393-8. https://doi.org/10.1038/ nature 11622 .

5. Swindle MM, Makin A, Herron AJ, Clubb FJ Jr, Frazier KS. Swine as models in biomedical research and toxicology testing, (in eng). Vet Pathol. 2012;49(2):344-56. https://doi.org/10.1177/0300985811402846.

6. Schook LB, et al. DNA-based animal models of human disease: from genotype to phenotype, (in eng). Dev Biol (Basel). 2008;132:15-25. https://doi.org/10.1159/00031 7140 .

7. Schachtschneider KM, et al. The oncopig cancer model: an innovative large animal translational oncology platform, (in eng). Front Oncol. 2017;7:190. https://doi.org/ 10.3389/fonc.2017.00190.

8. Schachtschneider KM, Madsen O, Park C, Rund LA, Groenen MA, Schook LB. Adult porcine genome-wide DNA methylation patterns support pigs as a biomedical model, (in eng). BMC Genomics. 2015;16:743. https:// doi.org/10.1186/s12864-015-1938-x.

9. Choi M, et al. Genome-wide analysis of DNA methylation in pigs using reduced representation bisulfite sequencing, (in eng). DNA Res. 2015;22(5):343-55. https://doi.org/10. 1093/dnares/dsv017.

10. Schook LB, Collares TV, Darfour-Oduro KA, De AK, Rund LA, Schachtschneider KM, Seixas FK. Unraveling the swine genome: implications for human health. Annu Rev Anim Biosci. 2015;3:219-44. https://doi.org/10. 1146/annurev-animal-022114-110815.

11. Gutierrez K, Dicks N, Glanzner WG, Agellon LB, Bordignon V. Efficacy of the porcine species in biomedical research. Front Genet. 2015;6:293.

12. Santulli G, et al. "Models for preclinical studies in agingrelated disorders: one is not for all," (in eng), Translational medicine @ UniSa. 2016;13,pp. 4-12. [Online]. Available: https://pubmed.ncbi.nlm.nih.gov/27042427. https:// www.ncbi.nlm.nih.gov/pmc/articles/PMC4811343/.

13. N. C. Ganderup, W. Harvey, J. T. Mortensen, and W. Harrouk, "The minipig as nonrodent species in toxicology-where are we now?," 2012 Nov-Dec, 2012. [Online]. Available: http://europepmc.org/abstract/MED/23134714. https://doi.org/10.1177/1091581812462039.

14. Heino TJ, Alm JJ, Moritz N, Aro HT. Comparison of the osteogenic capacity of minipig and human bone marrow-derived mesenchymal stem cells. J Orthop Res. 2012;30(7):1019-25.

15 Schwartz RS, et al. Drug-eluting stents in preclinical studies: updated consensus recommendations for preclinical evaluation. Circ Cardiovasc Interv. 2008;1(2):143-53.

16. Coronel R, et al. Dietary n-3 fatty acids promote arrhythmias during acute regional myocardial ischemia in isolated pig hearts. Cardiovasc Res. 2007;73(2):386-94.

17 Dixon JA, Spinale FG. Large animal models of heart failure: a critical link in the translation of basic science to clinical practice. Circ Heart Fail. 2009;2(3):262-71.

18. Ekeløf S, Rosenberg J, Jensen JS, Gögenur I. Pharmacological attenuation of myocardial reperfusion injury in a closed-chest porcine model: a systematic review. J Cardiovasc Transl Res. 2014;7(6):570-80.

19. Al-Mashhadi RH, et al. Familial hypercholesterolemia and atherosclerosis in cloned minipigs created by DNA transposition of a human PCSK9 gain-of-function mutant. Sci Transl Med. 2013;5(166):166ra1.

20. Shim J, Al-Mashhadi RH, Sørensen CB, Bentzon JF. Large animal models of atherosclerosis-new tools for persistent problems in cardiovascular medicine. $\mathrm{J}$ Pathol. 2016;238(2):257-66.

21. Schook LB, Collares TV, Hu W, Liang Y, Rodrigues FM, Rund LA, Schachtschneider KM, Seixas FK, Singh K, Wells KD, Walters EM, Prather RS, Counter CM. A Genetic Porcine Model of Cancer. PLoS One. 2015;10(7):e0128864. https://doi.org/10.1371/journal. pone.0128864.

22. Wolf E, Braun-Reichhart C, Streckel E, Renner S. Genetically engineered pig models for diabetes research," (in eng). Transgenic Res. 231:27-38, 2014/02// 2014. https://doi.org/10.1007/s11248-013-9755-y.

23. Gaba RC, et al. Development and comprehensive characterization of porcine hepatocellular carcinoma for translational liver cancer investigation, (in eng). Oncotarget. 2020;11(28):2686-701. https://doi.org/10.18632/ oncotarget.27647.

24. Kalla D, Kind A, Schnieke A. Genetically engineered pigs to study cancer, (in eng). Int J Mol Sci. 2020:21 2. https://doi.org/10.3390/ijms21020488.

25. Al-Mashhadi RH, et al. Diabetes with poor glycaemic control does not promote atherosclerosis in genetically modified hypercholesterolaemic minipigs. Diabetologia. 2015;58(8):1926-36.

26. Elmadhun NY, Lassaletta AD, Chu LM, Sellke FW. Metformin alters the insulin signaling pathway in ischemic cardiac tissue in a swine model of metabolic syndrome. J Thorac Cardiovasc Surg. 2013;145(1):258-66.

27. Schachtschneider KM, et al. A validated, transitional and translational porcine model of hepatocellular carcinoma, (in eng). Oncotarget. 2017;8(38):63620-34. https://doi.org/10.18632/oncotarget.18872.

28. Horvath S. DNA methylation age of human tissues and cell types, (in eng). Genome Biol. 2013;14(10):R115. https://doi.org/10.1186/gb-2013-14-10-r115.

29. Horvath S, Levine AJ. HIV-1 infection accelerates age according to the epigenetic clock, (in eng). J Infect Dis. 2015;212(10):1563-73. https://doi.org/10.1093/infdis/ jiv277.

30. Horvath S, et al. Obesity accelerates epigenetic aging of human liver. Proc Natl Acad Sci USA. 2014;111(43):1553843. https://doi.org/10.1073/pnas.1412759111.

31 Horvath S, Raj K. DNA methylation-based biomarkers and the epigenetic clock theory of ageing, (in eng). Nat Rev Genet. 2018. https://doi.org/10.1038/ s41576-018-0004-3.

32. Marioni R, et al. DNA methylation age of blood predicts all-cause mortality in later life. Genome Biol. 2015;16 1:25. [Online]. Available: http://genomebiology.com/ 2015/16/1/25.

33. Chen $\mathrm{BH}$, et al. DNA methylation-based measures of biological age: meta-analysis predicting time to death, 
(in Eng). Aging (Albany NY). 2016;8(9):1844-65. https://doi.org/10.18632/aging.101020.

34. Lu AT, et al. DNA methylation GrimAge strongly predicts lifespan and healthspan, (in eng). Aging (Albany NY). 2019;11(2):303-27. https://doi.org/10.18632/aging. 101684.

35. Morgello S, et al. The National NeuroAIDS Tissue Consortium: a new paradigm in brain banking with an emphasis on infectious disease. Neuropathol Appl Neurobiol. 2001;27(4):326-35.

36. Horvath $\mathrm{S}$, et al. Perinatally acquired HIV infection accelerates epigenetic aging in South African adolescents, (in eng). AIDS (London, England). 2018;32(11):1465-74. https://doi.org/10.1097/QAD.0000000000001854.

37. Kabacik S, Horvath S, Cohen H, Raj K. Epigenetic ageing is distinct from senescence-mediated ageing and is not prevented by telomerase expression, (in eng). Aging (Albany NY). 2018;10(10):2800-15. https://doi.org/10. 18632/aging.101588.

38. Arneson A, et al. A mammalian methylation array for profiling methylation levels at conserved sequences. bioRxiv. 2021:2021.01.07.425637. https://doi.org/10.1101/2021. 01.07.425637.

39. Zhou W, Triche TJ Jr, Laird PW, Shen H. SeSAMe: reducing artifactual detection of DNA methylation by Infinium BeadChips in genomic deletions. Nucleic Acids Res. 2018;46(20):e123-e123. https://doi.org/10.1093/nar/ gky691.

40. de Magalhaes JP, Costa J, Church GM. An analysis of the relationship between metabolism, developmental schedules, and longevity using phylogenetic independent contrasts, (in eng). J Gerontol A Biol Sci Med Sci. 2007;62(2):149-60.

41. de Magalhaes JP, Costa J, Toussaint O. HAGR: the Human Ageing Genomic Resources. Nucleic Acids Res. 2005;33. no. Database issue, pp. D537-43. https://doi.org/ 10.1093/nar/gki017.

42. Weigl R. Longevity of mammals in captivity; from the living collections of the world. 2005.

43. Friedman J, Hastie T, Tibshirani R. Regularization paths for generalized linear models via coordinate descent. J Stat Softw. 2010;33(1):1-22.

44. Langfelder P, Horvath S. WGCNA: an R package for weighted correlation network analysis. BMC Bioinformatics. 2008;9 1:559. [Online]. Available: http://www.biome dcentral.com/1471-2105/9/559.

45. McLean CY, et al. GREAT improves functional interpretation of cis-regulatory regions. Nat Biotechnol. 2010;28:2010. https://doi.org/10.1038/nbt.1630.

46. Bailey TL, et al. MEME Suite tools for motif discovery and searching. Nucleic Acids Res. 2009;37(suppl_2):W202-8. https://doi.org/10.1093/nar/ gkp335.

47 Wege H, Muller A, Muller L, Petri S, Petersen J, Hillert C. Regeneration in pig livers by compensatory hyperplasia induces high levels of telomerase activity. Comp Hepatol. 2007;6:6. https://doi.org/10.1186/1476-5926-6-6.

48. Ito T, Teo YV, Evans SA, Neretti N, Sedivy JM. Regulation of cellular senescence by polycomb chromatin modifiers through distinct DNA damage- and histone methylation-dependent pathways. Cell Rep. 2018;22(13):3480-92. https://doi.org/10.1016/j.celrep. 2018.03.002.

49. Yengo L, et al. Meta-analysis of genome-wide association studies for height and body mass index in approximately 700000 individuals of European ancestry. Hum Mol Genet. 2018;27(20):3641-9. https://doi.org/10.1093/hmg/ ddy271.

50. He J, et al. PKD1 mono-allelic knockout is sufficient to trigger renal cystogenesis in a mini-pig model, (in eng). Int J Biol Sci. 2015;11(4):361-9. https://doi.org/10.7150/ ijbs.10858.

51. Vodicka $\mathrm{P}$, et al. The miniature pig as an animal model in biomedical research, (in eng). Ann N Y Acad Sci. 2005;1049:161-71. https://doi.org/10.1196/annals.1334.015.

52. Lu AT, et al. Universal DNA methylation age across mammalian tissues. bioRxiv. 2021:2021.01.18.426733. https:// doi.org/10.1101/2021.01.18.426733.

53. Stocks T, et al. TFAP2B influences the effect of dietary fat on weight loss under energy restriction, (in eng). PLoS ONE. 2012;7(8):e43212. https://doi.org/10.1371/journal. pone. 0043212 .

54. Nambu H, et al. Characterization of metabolic phenotypes of mice lacking GPR61, an orphan G-protein coupled receptor, (in eng). Life Sci. 2011;89(21-22):765-72. https://doi.org/10.1016/j.lfs.2011.09.002.

55. Felix JF, et al. Genome-wide association analysis identifies three new susceptibility loci for childhood body mass index, (in eng). Hum Mol Genet. 2016;25(2):389-403. https://doi.org/10.1093/hmg/ddv472.

56. Goni L, et al. Interaction between an ADCY3 genetic variant and two weight-lowering diets affecting body fatness and body composition outcomes depending on macronutrient distribution: a randomized trial, (in eng). Nutrients. 2018;10 6. https://doi.org/10.3390/nu10060789.

57. Grarup N, et al. Loss-of-function variants in ADCY3 increase risk of obesity and type 2 diabetes, (in eng). Nat Genet. 2018;50(2):172-4. https://doi.org/10.1038/ s41588-017-0022-7.

58. Saeed S, et al. Loss-of-function mutations in ADCY3 cause monogenic severe obesity, (in eng). Nat Genet. 2018;50(2):175-9. https://doi.org/10.1038/ s41588-017-0023-6.

59. Kaewsutthi S, Santiprabhob J, Phonrat B, Tungtrongchitr A, Lertrit P, Tungtrongchitr R. Exome sequencing in Thai patients with familial obesity, (in eng). Genet Mol Res. 2016;15 2. https://doi.org/10.4238/gmr.15028311.

60. Hoffe B, Holahan MR. The use of pigs as a translational model for studying neurodegenerative diseases, (in eng). Front Physiol. 2019;10:838. https://doi.org/10.3389/fphys. 2019.00838.

61. Crisóstomo V, et al. Common swine models of cardiovascular disease for research and training, (in eng). Lab Anim (NY). 2016;45(2):67-74. https://doi.org/10.1038/laban. 935.

62. Levine ME, et al. Menopause accelerates biological aging, (in eng). Proc Natl Acad Sci USA. 2016;113(33):932732. https://doi.org/10.1073/pnas.1604558113.

Publisher's note Springer Nature remains neutral with regard to jurisdictional claims in published maps and institutional 
affiliations. 